\title{
Clase, política y percepción del conflicto de clases en Chile*
}

\author{
Class, politics and perception of class conflict in Chile
}

Classe, política e percepção do conflito de classes no Chile

\author{
Pablo Pérez Ahumada** , Valentina Andrade de la Horra***
}

\section{RESUMEN}

En la última década, Chile ha experimentado un renacer de la

Palabras clave:

política contenciosa, cuyo punto más alto se observó durante

Chile, clases,

el Estallido Social de octubre de 2019. Una serie de investiga-

conflicto,

ciones han estudiado la revitalización de las protestas en Chile, mostrando cómo ellas son el resultado del malestar generado empresarios, política, por los altos niveles de desigualdad socioeconómica y de poder trabajadores. del país. A pesar de esto, pocas investigaciones han analizado cómo los chilenos y chilenas perciben el conflicto entre clases. En este artículo estudiamos cómo las percepciones de conflicto entre empresarios y trabajadores varían según la clase social, el nivel de privación económica percibido y la identificación con el continuo izquierda-derecha. Los hallazgos indican que estos tres factores afectan significativamente las percepciones de conflicto de clases. Por ejemplo, ser un trabajador o trabajadora no calificada e identificarse con la izquierda refuerza significativamente la percepción de oposición de clase. Nuestros análisis sugieren que la percepción de conflicto también se ve reforzada por el hecho de no identificarse con el continuo izquierda-derecha. Al

\footnotetext{
* Este estudio fue parcialmente financiado por el Centro de Estudios del Conflicto y la Cohesión Social (COES; ANID/ FONDAP/15130009) y por el Proyecto Fondecyt No 11190229 "Determinantes institucionales y políticos del conflicto entre empresarios y trabajadores: los casos de Argentina y Chile en perspectiva comparada".

** Chileno. Doctor en Sociología, Universidad de California - San Diego, Estados Unidos. Profesor Asistente del Departamento de Sociología, Universidad Alberto Hurtado e Investigador Adjunto del Centro de Estudios del Conflicto y Cohesión Social (COES). Santiago de Chile, Chile.pperez@uahurtado.cl

*** Chilena. Licenciada en Sociología, Universidad de Chile. Estudiante del Magíster en Economía, Pontificia Universidad Católica y Becaria del Centro de Estudios del Conflicto y Cohesión Social (COES). Santiago de Chile, Chile. valentinaandrade@uchile.cl
} 
final del artículo ofrecemos una explicación de estos hallazgos y reflexionamos acerca de sus implicancias para el estudio del conflicto político y de clases en el país.

\begin{abstract}
In the last decade, Chile has experienced a revival of contentious politics. We witnessed its highest point during the October 2019 Social Outburst. Several investigations have studied the revitalization of protests in Chile, showing how they result from the discontent generated by the country's high levels of socioeconomic and power inequality. Despite this, few studies have examined how Chileans perceive the conflict between classes. This article studies how perceptions of conflict between employers and workers vary according to social class, perceived level of economic deprivation, and left-right identity. The findings indicate that these three factors significantly affect perceptions of class conflict. For example, being an unskilled worker and identifying with the left significantly reinforces the perception of class opposition. Our analyses suggest that the perception of conflict is also reinforced by not identifying with the left-right spectrum. At the end of the article, we explain these findings and reflect on their implications for the study of political and class conflict in the country.
\end{abstract}

\section{RESUMO}

Na última década, o Chile experimentou um renascer da política contenciosa, cujo ápice se observou durante a Revolta Social de outubro de 2019. Uma série de pesquisas estudaram a revitalização dos protestos no Chile, mostrando como são o resultado do malestar gerado pelos elevados níveis de desigualdade socioeconômica e de poder do país. Apesar disto, poucos estudos analisaram como os chilenos e as chilenas percebem o conflito entre classes. Neste artigo, estudamos como as percepções de conflito entre empresários e trabalhadores variam segundo a classe social, o nível de privação econômica percebido e a identificação com o contínuo esquerda-direita. Os achados indicam que estes três fatores afetam significativamente as percepções de conflito de classes. Por exemplo, ser um trabalhador ou trabalhadora não qualificada e identificada com a esquerda reforça a percepção de oposição de classe de forma significativa. Nossas análises sugerem que a percepção de conflito também se vê reforçada pelo fato de não se identificar com o contínuo esquerda-direita. Ao final do artigo, oferecemos uma explicação para estes achados e refletimos sobre suas implicações para o estudo do conflito político e de classes no país.
Keywords: Chile, class, conflict, employers, politics, workers.

Palavraschave: Chile, classes, conflito, empresários, política, trabalhadores. 


\section{Introducción}

A inicios de la década de 2010, Chile experimentó un renacer de la "política contenciosa" (Donoso y Von Bülow, 2017) cuyo punto más alto se observó durante el Estallido Social del 18 de octubre de 2019 (Somma et al., 2020). En los últimos años, diversas investigaciones han analizado las causas del incremento de la movilización social en Chile. Gran parte de ellas ha ofrecido explicaciones que enfatizan los altos niveles de desigualdad socioeconómica y de poder observados en Chile, así como el distanciamiento entre la ciudadanía y actores políticoinstitucionales, tales como partidos y élites políticas (Joignant et al., 2017; Luna, 2016; Programa de las Naciones Unidas para el Desarrollo [PNUD], 2015; Somma, 2017). A pesar de esto, pocas investigaciones han analizado sistemáticamente cómo se percibe el conflicto de clases en Chile, y cómo dichas percepciones se ven afectadas por la clase social y la identificación política de las personas.

En este artículo desarrollamos un estudio de este tipo a través de una investigación empírica, cuantitativa, centrada en el concepto de política de clases. Dicho concepto es utilizado para analizar cómo los clivajes de clase se traducen en la polarización de percepciones y actitudes y, a partir de ello, en la movilización política por parte de actores que representan (y a su vez forman) intereses de clase (Weakliem y Adams, 2011). De modo más específico, siguiendo estudios recientes acerca de percepciones y conflicto de clase (Dodson, 2017; Edlund y Lindh, 2015; Robison y Stubager, 2018; Wright, 2015), en este artículo estudiamos cómo la clase social -entendida como posición objetiva en las relaciones de producción y como un fenómeno subjetivo expresado en identidades de clase- así como las percepciones de privación económica y las identificación con el continuo izquierda-derecha configuran las percepciones del conflicto entre clases sociales.

A partir de datos de la Encuesta del Centro de Estudios del Conflicto y Cohesión Social, COES (2014), construimos una escala de percepción del conflicto de clases compuesta por ítems que miden, de modo similar a la "escala de conciencia de clase" de Erik O. Wright (1997), en qué medida las personas encuestadas están de acuerdo con que el bajo nivel de vida de los trabajadores es consecuencia de las acciones de los empresarios. Siguiendo los criterios generales del modelo de clases de Wright (1997), diversos análisis de regresión nos permiten 
demostrar la importancia de la clase social, la cual se expresa en que quienes se encuentran en una posición subordinada en las relaciones de producción (por ejemplo, trabajadores y trabajadoras no calificadas o autoempleados informales) y quienes se identifican con la clase "baja" perciben más conflicto de clases que aquellos que se ubican en una posición privilegiada (empresarios y expertos directivos) o que se identifican con la clase alta. Asimismo, nuestros hallazgos indican que la percepción de privación económica y la identificación con la izquierda refuerzan de modo significativo la percepción de dicho conflicto. A partir de estos y otros resultados (por ejemplo, quienes no se identifican con el continuo izquierda-derecha también perciben fuertes conflictos de clase), este artículo ofrece una explicación general acerca de cómo opera la política de clases en Chile. Entre otras cosas, notamos cómo la "politización de la desigualdad" destacada en la literatura reciente y comúnmente asociada con la irrupción de la protesta social sigue ocurriendo en un contexto de fuerte distanciamiento entre movimientos sociales y partidos políticos tradicionales. Ello hace que el malestar de clase sea muchas veces "no político", en el sentido de que no es el resultado de la articulación de partidos políticos con agendas explícitas de clase.

\section{Política de clases y conflicto social en Chile: causas y consecuencias de la movilización social durante la década de 2010}

Tanto la historia sociopolítica de Chile como acontecimientos más recientes, en particular, los asociados al Estallido Social, sugieren que Chile es un buen caso para estudiar la relación entre desigualdad de clases y conflicto social. Como se sabe, la instalación de la dictadura militar (1973-1990) implicó que el poder del movimiento sindical, el cual fue construido por décadas a través de la vinculación con partidos de izquierda (Angell, 1972), haya sido mermado a través de la represión estatal y políticas económicas neoliberales. Estos cambios políticoeconómicos debilitaron severamente a los sectores más activos de la clase trabajadora, por ejemplo, a trabajadores industriales empleados en empresas nacionales orientadas al mercado interno (Drake, 1996). Así, con el retorno a la democracia, el movimiento sindical siguió estando en una posición debilitada. Más aún, los partidos gobernantes 
de centroizquierda agrupados en la Concertación abandonaron su estrategia de organización de los sectores populares. De ese modo, con el fin de proteger la estabilidad del pacto de transición entre "entre elites", estos partidos no solo evitaron hacer transformaciones significativas al marco institucional heredado de la dictadura, sino que también optaron por desmovilizar "por arriba" a los movimientos sociales que se habían organizado en los últimos años de la dictadura (Donoso, 2016; Luna, 2016; Roberts, 2017; Somma y Medel, 2017). Esto reforzó la debilidad de los movimientos sociales y de trabajadores durante las primeras décadas de gobiernos posdictatoriales.

Sin embargo, este escenario comenzó a cambiar a fines de la década de 2010 y, especialmente, luego de 2011. Desde entonces, Chile ha sido testigo de un renacer de la "política contenciosa", el cual se ha expresado en la aparición de movimientos sociales de diversa índole (sindicales, estudiantiles y feministas, entre otros) que, a través de protestas masivas, han exigido transformaciones institucionales profundas en el país (Donoso y Von Bülow, 2017; Somma y Medel, 2017). La expresión más reciente de esto son las protestas masivas iniciadas el 18 de octubre de 2019, en las cuales millones de personas se tomaron las calles exigiendo no solo mejoras sustanciales en las condiciones de vida, sino también cambios profundos en el modelo económico - por ejemplo, el fin de la privatización de servicios sociales- y en el régimen político (particularmente, el fin de la constitución impuesta en 1980 por la dictadura) (Santibáñez, 2019; Unidad Social, 2019).

Diversas investigaciones recientes han analizado las causas de este renacer de la movilización social en Chile. De una manera u otra, todas estas investigaciones han ofrecido explicaciones que enfatizan los altos niveles de desigualdad socioeconómica y de poder del país. Algunos analistas señalan, por ejemplo, que el aumento de la movilización social observado desde inicios de la década de 2010 se explica por el progresivo distanciamiento entre movimientos sociales y actores político-institucionales como partidos políticos, gobierno y élites políticas (Garretón, 2016). Según este argumento, dicho distanciamiento impidió el desarrollo de un sistema político más representativo, capaz de canalizar por medios institucionales los altos niveles de desigualdad del país (Somma y Medel, 2017). Otros estudios han puesto el énfasis en cómo la persistencia de desigualdades econó- 
micas y de poder entre clases creó fuertes sentimientos de malestar y descontento en gran parte de la población chilena (Joignant et al., 2017; Luna, 2016; PNUD, 2015; Somma, 2017).

A partir de este tipo explicaciones, los estudios recientes coinciden en señalar que el renacer de la protesta social en Chile ha tenido un profundo impacto tanto a nivel individual como sobre el sistema político e institucional. A nivel individual, las protestas han repolitizado a los chilenos (PNUD, 2015; Roberts, 2017). Según el informe Desarrollo humano en Chile: Los tiempos de la politización, del PNUD (2015), desde 2011 los chilenos no solo están más dispuestos a asistir a manifestaciones públicas y a participar en otras formas "no convencionales" de involucramiento político, sino que también tienen más disposición para resolver cuestiones redistributivas a través de instancias deliberativas y no tecnocráticas. Por su parte, a nivel político-institucional, las protestas han reforzado la discusión pública sobre la desigualdad y, de ese modo, han repolitizado ("des-tecnocratizado") el debate en torno a la política social (Donoso, 2016; Roberts, 2017).

En línea con esto, tanto investigaciones empíricas como análisis no académicos han señalado que el gran empresariado ha sido uno de los grupos sociales que más ha perdido legitimidad como resultado de las movilizaciones sociales. Datos de la Encuesta Latinobarómetro sugieren que en Chile el nivel de confianza en las grandes empresas ha experimentado reducciones sustanciales en los últimos años. La Figura 1 muestra que entre 2001 y 2010 el porcentaje promedio de chilenos que confiaba en las grandes empresas fue de 40\% (más aún, en 2001, 2005 y 2010 el porcentaje alcanzó el 44\%). En contraposición a esa tendencia, dicho porcentaje bajó a 33\% en 2015 e incluso llegó a un valor de $30 \%$ en 2011. El caso de Chile contrasta con el de países como Uruguay, donde desde 2006 el nivel de confianza ha permanecido estable alrededor de aproximadamente $47 \%$. Chile también difiere de casos como el argentino y el ecuatoriano. A pesar de haber niveles de confianza promedio menores, en estos países la confianza en los empresarios ha tendido a crecer desde 2007, llegando incluso al 41\% en 2011 (Argentina) y a casi 50\% en 2015 (Ecuador). 
Figura 1

Confianza en grandes empresas en 10 países de América Latina (2001-2015)

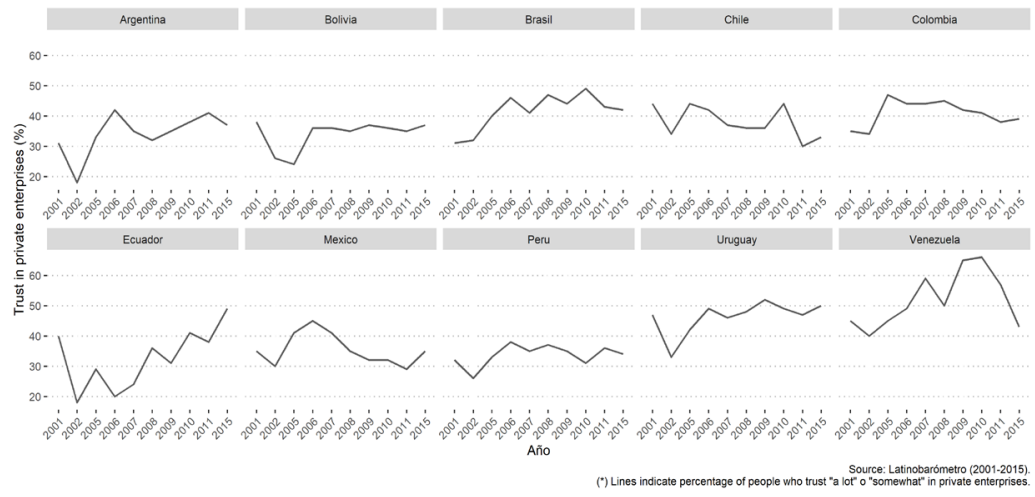

Fuente: Latinobarómetro (2001-2015).

A la luz de este tipo de fenómenos, diversos analistas han afirmado que las movilizaciones sociales iniciadas en 2011 han ayudado a reforzar el tradicional clivaje entre izquierda y derecha en Chile, aun cuando la acción colectiva ha sobrepasado con creces los márgenes de los partidos tradicionales (Donoso y Von Bülow, 2017; Roberts, 2017). Esto supondría una reversión de la tendencia mostrada en otros estudios anteriores, que sostienen que a lo largo de la década del 2000 el clivaje izquierda-derecha perdió importancia como variable definitoria de las preferencias redistributivas de chilenos y chilenas (Castillo et al., 2013).

Estos fenómenos han sido claves para explicar los procesos políticos iniciados el 18 de octubre de 2019. A pesar de que la intensidad de las protestas y sus efectos (por ejemplo, la instalación de un Convención Constituyente) superaron todos los eventos observados durante la década de 2010, dichos fenómenos son inseparables de un proceso más largo de acumulación de malestares sociales y de politización del debate sobre desigualdad (Somma et al., 2020). El ejemplo más claro de esto ocurrió luego del ciclo de movilizaciones de octubre de 2019. En el plebiscito por una nueva constitución del 25 de octubre de 2020, el 78\% del país aprobó una nueva constitución y el 79\% de los y las votantes señaló que ella debería ser redactada por una convención constituyente, es decir, una asamblea compuesta enteramente de ciudadanos. Resultados similares fueron observados durante las elecciones 
municipales y de delegados constituyentes del 15-16 de mayo de 2021. Dichas elecciones han sido definidas como un "Estallido Electoral" (Cortés, 2021), no solo porque resultaron en la derrota estrepitosa de los partidos de derecha y de centroizquierda tradicional (exConcertación), sino que también porque ellas dieron cuenta de la revitalización de la izquierda antineoliberal (representada por el Partido Comunista y el Frente Amplio) y de la emergencia de una poderosa (pero aún heterogénea y contradictoria) "Lista del Pueblo".

Estos fenómenos son recientes y aún tienen que ser estudiados de modo sistemático. A pesar de esto, la investigación empírica de los últimos años ha entregado elementos para entender las causas de la protesta social y sus consecuencias políticas. En este sentido, dicha investigación ha representado un avance muy relevante para el estudio del conflicto social en el país. Sorprendentemente, a pesar de que la desigualdad de clases es comúnmente considerada como una de las causas principales de las protestas (y del Estallido Social en particular), pocas investigaciones han puesto al conflicto de clases en el centro del análisis. Esto es aún más notorio cuando se trata de investigaciones cuantitativas acerca del conflicto de clases. Dos excepciones a esta tendencia son los estudios desarrollados en los últimos años por Pérez (2014) y Castillo et al. (2015). En línea con la investigación internacional, Pérez (2014) demuestra que quienes se ubican en la clase trabajadora o tienen un origen de clase trabajadora perciben más oposición entre trabajadores y empresarios que quienes se encuentran en una posición o tienen un origen de clase privilegiado. De igual manera, Castillo et al. (2015) muestran que el nivel de conflicto de clases percibido por las personas se ve afectado por variables demográficas y socioeconómicas (por ejemplo, hombres y personas de mayores ingresos tienden a percibir menos conflicto) y, especialmente, por las opiniones que las personas tienen respecto de la situación general del país.

En este artículo contribuimos a este tipo de investigaciones analizando cómo fenómenos asociados a la clase social y al comportamiento político afectan las percepciones del conflicto de clases. De modo más concreto, a partir de la investigación internacional reciente de clases sociales y percepciones de desigualdad y conflicto (ver abajo), estudiamos si la clase social (medida como posición de clase 
y autoidentificación de clase), las experiencias de desigualdad resultantes de una posición desaventajada en la estructura social (privación económica) y la identificación con el continuo izquierda-derecha generan variaciones significativas en las percepciones del conflicto de clases. Junto con esto, pretendemos contribuir a la investigación reciente presentando hallazgos basados en una medición novedosa del conflicto de clases percibido. Estudios previos han estudiado estas percepciones a partir de escalas que miden actitudes centradas en el ámbito laboral Pérez (2014), o bien, percepciones generales del nivel de conflicto entre empresarios/ricos y trabajadores/pobres (Castillo et al., 2015). De forma complementaria a estos estudios, en este artículo construimos una escala de percepciones de conflicto de clases formada por una serie de enunciados referidos al modo en que la relación entre empresarios y trabajadores tiene efectos concretos sobre la desigualdad. Tal como se detalla en la sección metodológica, la escala se compone de ítems que miden, entre otras cosas, en qué medida las personas encuestadas están de acuerdo con que la desigualdad o el bajo nivel de vida de los trabajadores son consecuencia de las acciones de los empresarios. En este sentido, nuestra escala recoge alguno de los aspectos centrales de la "escala de conciencia de clase" propuesta por Wright (1997).

La sección siguiente presenta una breve revisión de la literatura relevante para entender cómo fenómenos asociados a la clase y al comportamiento político configuran las percepciones de conflicto entre clases. Junto con mostrar cómo dicha literatura ha analizado problemas similares al estudiado acá, en esta sección se presentan las hipótesis que dirigieron nuestra investigación. Luego de ello, las secciones siguientes describen los datos y métodos utilizados en este trabajo, así como los resultados. Finalmente, en la última sección se describen las conclusiones e implicancias de nuestro análisis.

\section{Revisión de la literatura e hipótesis}

Posición de clase, identidad de clase y sus efectos sobre las percepciones de conflicto

La literatura referente a las percepciones de clase ha desarrollado uno de los argumentos más explícitos en cuanto a los efectos subjeti- 
vos de la clase social (Evans, 1992; Robison y Stubager, 2018; Wright, 1997). Basándose en conceptos inspirados en el marxismo, Wright (1997) estudió los patrones de conciencia de clase en varios países desarrollados. El autor define la conciencia de clase como la toma de conocimiento de los individuos acerca de sus intereses materiales, es decir, sus intereses en relación con el bienestar económico y el poder derivado de su posición en las relaciones de explotación (Wright, 1989). A partir de dicha definición, Wright (1997) construyó una "escala de conciencia de clase" (p. 407) y demostró que las personas con una posición de clase trabajadora poseían una "conciencia anticapitalista" (p. 411) significativamente más fuerte que quienes se ubicaban en la burguesía o en la clase media. Este autor explicó estos resultados señalando que los trabajadores son, en tanto miembros de la clase explotada y dominada en las relaciones de producción, más propensos a tener intereses anticapitalistas que los explotadores (capitalistas) o quienes se encuentran en "posiciones contradictorias" de clase (es decir, individuos de clase media que ocupan simultáneamente roles de explotados y explotadores en las relaciones de producción). Otros estudios han llegado a conclusiones similares a las de Wright. Estos han demostrado que la posición de la clase de los individuos es, al denotar su lugar en las relaciones de producción, un determinante relevante de la identidad de clase, de sus intereses de clase (por ejemplo, sus preferencias redistributivas) y de sus percepciones de conflicto clases (Dodson, 2017; Edlund y Lindh, 2015; Elbert y Pérez, 2018; Langsæther y Evans, 2020; Robison y Stubager, 2018). Por ejemplo, Dodson (2017) ha demostrado que quienes pertenecen a la clase trabajadora tienen más probabilidades de apoyar políticas redistributivas vía impuestos que quienes pertenecen a la clase de profesionales y ejecutivos, mientras que en su estudio de una veintena de países desarrollados Edlund y Lindh (2015) concluyen que las personas de clase trabajadora perciben más conflicto de clases que las personas de clase privilegiada.

Como complemento de este tipo de investigaciones centradas en la posición de clases, otros analistas han afirmado que la clase social se manifiesta no solo como posición "objetiva" en las relaciones de producción, sino que también en términos "subjetivos", es decir, como identidad de clase (Fantasia, 1988; Thompson, 1966). En socio- 
logía, la identidad de clase ha sido muchas veces estudiada a través de modelos estadísticos que la analizan como "variable dependiente", es decir, que estudian cómo diversos factores sociodemográficos afectan la autoidentificación de las personas con una clase en particular (Andersen y Curtis, 2012; Elbert y Pérez, 2018; Hout, 2008; Oddsson, 2018; Vanneman y Cannon, 1987). Sin embargo, al notar cómo la autoidentificación de clase expresa procesos subjetivos a través de los cuales las personas se definen así mismas como parte de una clase, diversos autores han afirmado que esta es un factor - esto es, una "variable independiente" - que refuerza los intereses sociopolíticos de las personas. Wright (1997), por ejemplo, afirma que la autoidentificación de clase permite que las personas tengan conciencia de su posición en las relaciones de producción (particularmente, su posición desaventajada para el caso de las personas de clase trabajadora). Por eso, señala el autor, cuando la identidad de clase es fuerte, existe mayor conciencia de los intereses de clase. En línea con esto, Robison y Stubager (2018) demuestran que la identificación con la clase trabajadora refuerza significativamente la conciencia de la oposición entres clases.

A partir de lo anterior se pueden plantear dos hipótesis acerca del impacto que podría tener la posición de clase y la autoidentificación de clase en la percepción del conflicto de clases:

$\mathrm{H}_{1,1}$ : Quienes se ubican en posiciones de clase trabajadora o de clase subordinada están sujetos a relaciones de explotación y dominación en el seno de la producción. Esto los hace percibir mayor conflicto de clase que quienes se ubican en posiciones de clase privilegiadas.

$\mathrm{H}_{1.2}$ : Quienes tienen una autoidentificación de clase trabajadora o "baja" se perciben a sí mismos como parte de una clase ubicada en una posición "desaventajada" en las relaciones de producción. Por eso, perciben más conflicto de clases que quienes se identifican con las clases privilegiadas.

\section{Privación económica y percepciones de desigualdad}

De modo complementario a la literatura en torno a las clases sociales, diversas investigaciones han estudiado los factores que determinan cómo las personas perciben la desigualdad social. Si bien en décadas 
pasadas algunos estudios sugirieron que los niveles de conciencia del antagonismo entre clases podrían ser bajos (Kelley y Evans, 1995; Kluegel y Smith, 1986), ellos han demostrado que quienes están en posiciones más desaventajadas social y económicamente tienen más probabilidades de sostener actitudes críticas frente a la desigualdad y de respaldar políticas redistributivas, en comparación con quienes no lo están (Alesina y Giuliano, 2011; Burak, 2013; Hadler, 2005; Kelley y Evans, 2017; Kreidl, 2000; Reynolds y Xian, 2014; Svallfors, 2006). Mientras algunos analistas han explicado estos hallazgos a través de nociones como autointerés (Kluegel y Smith, 1986) o economía moral (Svallfors, 2006), otros han enfatizado el concepto de privación económica (economic deprivation) (Hadler, 2005; Kreidl, 2000; Robinson y Bell, 1978).

La idea de privación económica ha sido utilizada para describir experiencias de desigualdad resultantes de una posición desaventajada en el sistema de estratificación (Kreidl, 2000; Robinson y Bell, 1978). De modo similar al concepto de clase, la literatura distingue entre privación "absoluta" y "relativa" para diferenciar entre una situación de privación objetiva - por ejemplo, una posición desaventajada en el mercado laboral- y una situación de privación percibida "subjetivamente" (Kreidl, 2000, p. 156). El concepto de privación relativa ha ocupado un lugar importante en los estudios cuantitativos referidos a percepciones de la desigualdad. En estos, este concepto ha sido medido de diversas maneras. Algunos lo operacionalizan como oposición subjetiva entre lo que se gana y lo que se piensa que se debería ganar (Szirmai, 1986), o bien, como percepción individual del carácter "justo" o "merecido" del ingreso que se obtiene (Hadler, 2005; Robinson y Bell, 1978). Otros, por su parte, lo miden a partir de la evaluación subjetiva de si el ingreso que se obtiene alcanza o no para cubrir las necesidades del hogar (Kreidl, 2000). Más allá de estas diferencias, los resultados de estos estudios apuntan en la misma dirección: quienes se perciben a sí mismos en una situación de privación económica tienden a percibir más desigualdad o mayores brechas de ingreso que quienes se perciben como parte de un grupo privilegiado socioeconómicamente (Hadler, 2005; Kreidl, 2000; Robinson y Bell, 1978). Estas investigaciones no han examinado el tipo de variable dependiente analizada en este trabajo (nivel de conflicto de clases percibido). Sin embargo, han 
demostrado que la percepción de privación económica incrementa actitudes "críticas" hacia la riqueza, es decir, actitudes que enfatizan cómo la riqueza se explica más por aspectos estructurales del sistema de estratificación, que por el mérito individual de quienes la poseen (Kreidl, 2000). A partir de esto se puede plantear hipotéticamente que:

$\mathrm{H}_{2}$ : Quienes se perciben a sí mismos en una situación de privación económica percibirán mayor conflicto que quienes no se perciben en esa situación.

\section{Política de clases, autoidentificación política y percepciones de conflicto}

Además de los dos cuerpos teóricos ya revisados, una serie de investigaciones han analizado en qué medida las relaciones entre empresarios y trabajadores se ven afectadas por factores políticos. Mientras que las explicaciones de nivel macro han enfatizado cómo dichas relaciones se ven afectadas por variables tales como el tipo de vínculos existentes entre partidos de izquierda y movimiento sindical (Huber y Stephens, 2001; Kingstone et al., 2013; Korpi, 1985; Madrid, 2003; Murillo, 2001), enfoques de nivel micro han estudiado la manera en que las orientaciones político-ideológicas de las personas (por ejemplo, su identificación con el continuo izquierda-derecha) afecta sus percepciones y actitudes hacia fenómenos de desigualdad (Evans, 1997; Hadler, 2005; Kreidl, 2000).

Siguiendo este último enfoque, Castillo et al. (2013) estudiaron cómo el clivaje izquierda-derecha afecta las preferencias redistributivas de chilenos y chilenas. Los autores hicieron su medición a partir de la identificación individual con partidos políticos y la autoidentificación con el continuo izquierda-derecha. A partir de diversos análisis estadísticos, los autores demuestran que la identificación partidista tiene poco impacto sobre las preferencias redistributivas. Ellos explican este hallazgo como el resultado probable de la proximidad programática entre partidos de centroizquierda y derecha observada en Chile en el periodo estudiado por ellos (1999-2009). Junto con esto, los autores demuestran que la identificación individual con el continuo izquierda-derecha tiene un poder explicativo relativamente mayor que la identificación partidista. Sus modelos de regresión logística indican que quienes se identifican con la izquierda tienen más chances 
de apoyar medidas redistributivas en materia de impuestos, así como de encontrar injusta la desigualdad en salud y educación (Castillo et al., 2013). De modo similar, Evans (1997) y Kreidl (2000) presentan evidencia que demuestra que la identificación con la izquierda refuerza visiones estructurales - no meritocráticas - de la pobreza, la riqueza y de la desigualdad económica en general. Por su parte, Hadler (2005) muestra que quienes se posicionan a sí mismos en la izquierda del espectro político tienen actitudes más críticas hacia la desigualdad de ingresos que quienes se definen de derecha.

A partir de esto, entonces, se puede plantear hipotéticamente que:

$\mathrm{H}_{3}$ : Quienes se identifican con la izquierda política perciben mayor antagonismo de clases que quienes se identifican con la derecha o con el centro político.

\section{Datos y métodos}

En este artículo se utilizaron datos provenientes de una submuestra de la Encuesta COES aplicada en 2014, instrumento conducido en base a un diseño muestral multietápico con selección probabilística de casos en todas sus etapas. La encuesta es representativa de la población chilena entre 18 y 75 años, residente en las 22 ciudades principales del país (equivalentes al $78 \%$ de la población urbana y $68 \%$ de la población rural). La submuestra con la que se trabajó $(n=930)$ incluyó solo a la población ocupada, la cual pudo ser clasificada en clases sociales según el modelo marxista que acá ocupamos, inspirado en el esquema de Wright (1997).

Los datos utilizados fueron recolectados a mediados de la década del 2010. Por ello, es imposible asumir una relación causal entre las dinámicas develadas por estos datos y fenómenos ocurridos recientemente en el marco del Estallido Social. Sin embargo, debido al tipo de variables utilizadas - especialmente la variable dependiente (ver abajo) - creemos que estos datos sí son útiles para responder al objetivo de este artículo, a saber, analizar en qué medida la clase social y el posicionamiento político de las personas configuran sus percepciones en cuanto al conflicto de clases. Además, el hecho de que estos datos hayan sido recolectados antes del ciclo de protestas de 2019 no invalida, pensamos, el argumento central elaborado en las secciones 
siguientes. Este argumento indica que la clase social y la identificación con el continuo izquierda-derecha son determinantes significativos de las percepciones de conflicto. Más aún, creemos que estas conclusiones no solo siguen siendo válidas en la actualidad, sino que incluso podrían haberse reforzado luego de octubre de 2019, en la medida en que los eventos iniciados ese día han tendido a polarizar más los debates políticos respecto de clase, poder y desigualdad.

En este artículo, testeamos las hipótesis recién planteadas a partir de diversos modelos de regresión de mínimos cuadrados ordinarios. La variable dependiente de todos estos modelos de regresión - percepción del conflicto de clases- fue medida a través de una escala de percepción del conflicto de clases.

\section{Variable dependiente}

La escala de percepción del conflicto de clases fue construida a través de la suma simple de las respuestas a cuatro ítems tipo escala de Likert. Estos ítems midieron la actitud de los encuestados ante el conflicto entre empresarios y trabajadores, así como ante otros aspectos asociados al poder empresarial. La escala resultante tuvo rango de 0 a 16 puntos, en donde un mayor puntaje denota una percepción más elevada del conflicto entre clases, más específicamente, intereses de clase "antiempresariales" más fuertes. La Tabla 1 presenta detalles de los ítems utilizados para construir la escala e información descriptiva general. 
Tabla 1

Estadísticos descriptivos de la variable dependiente $(n=930)$

\begin{tabular}{|c|c|c|c|c|c|}
\hline $\begin{array}{l}\text { Ítems utilizados para } \\
\text { construir la escala ( } 0=\text { Muy } \\
\text { en desacuerdo; } 4 \text { = Muy de } \\
\text { acuerdo) }\end{array}$ & $\begin{array}{l}\text { Cargas } \\
\text { factoriales }\end{array}$ & Promedio & $\begin{array}{l}\text { Desviación } \\
\text { estándar }\end{array}$ & Mín. & Máx. \\
\hline $\begin{array}{l}\text { 1. Las grandes empresas } \\
\text { deberían hacer algo para } \\
\text { reducir las diferencias } \\
\text { salariales entre los } \\
\text { trabajadores de altos } \\
\text { salarios y aquellos con } \\
\text { bajos salarios. }\end{array}$ & 0,7773 & 3,220 & 0,849 & 0 & 4 \\
\hline $\begin{array}{l}\text { 2. El alto nivel de vida de } \\
\text { los dueños de las empresas } \\
\text { es resultado de lo poco que } \\
\text { pagan a sus trabajadores. }\end{array}$ & 0,8423 & 2,944 & 1,027 & 0 & 4 \\
\hline $\begin{array}{l}\text { 3. Una de las principales } \\
\text { razones de la alta } \\
\text { desigualdad en Chile es } \\
\text { que los dueños de las } \\
\text { empresas pagan poco a sus } \\
\text { trabajadores. }\end{array}$ & 0,8919 & 3,106 & 0,950 & 0 & 4 \\
\hline $\begin{array}{l}\text { 4. Los empresarios se } \\
\text { benefician a costa de los } \\
\text { consumidores. }\end{array}$ & 0,8425 & 3,187 & 0,885 & 0 & 4 \\
\hline $\begin{array}{l}\text { Escala de percepción del } \\
\text { conflicto de clases }\end{array}$ & & 12,456 & 3,115 & 0 & 16 \\
\hline \multicolumn{6}{|l|}{$\begin{array}{l}\text { (Autovalor, Factor } 1^{*}: 2,813 \text {; } \\
\text { Porcentaje de varianza } \\
\text { explicada: } 70,3 \% \text {; Alfa de } \\
\text { Cronbach: } 0,86 \text { ) }\end{array}$} \\
\hline $\begin{array}{l}\text { *Análisis factorial con métoc } \\
\text { Varimax). El factor } 1 \text { fue el ú }\end{array}$ & $\begin{array}{l}\text { de extracci } \\
\text { co con un a }\end{array}$ & $\begin{array}{l}\text { e compone } \\
\text { alor mayor }\end{array}$ & $\begin{array}{l}\text { tes principa } \\
1 .\end{array}$ & s (Rot & ción \\
\hline
\end{tabular}

Fuente: Elaboración propia.

La Tabla 1 muestra que el promedio de la escala es 12,5 puntos $(D E$ $=3,1$ ), lo cual sugiere que los niveles de conflicto de clase percibidos en Chile son altos. Por otro lado, los resultados del análisis factorial de componentes principales y del alfa de Cronbach $(\alpha=0,86)$ indican que la escala es unidimensional e internamente consistente.

\section{Variables independientes}

Las principales variables independientes de este artículo son tres: clase social (medida conjuntamente como posición objetiva y autoiden- 
tificación de clase), percepción de privación económica y, por último, identificación política.

\section{Clase social}

La clase social medida, en primer lugar, a partir la posición de clase de los encuestados. La posición de clase fue operacionalizada a través de una versión modificada de nueve categorías del esquema marxista propuesto por Wright (1997). Siguiendo estudios recientes para América Latina (Elbert y Pérez, 2018; Pérez, 2018), se dividió a la clase propietaria en tres categorías: pequeños empleadores (propietarios con 2 o más empleados); pequeña burguesía (trabajadores independientes con nivel de calificación profesional); y autoempleados informales (trabajadores independientes con educación terciaria incompleta o menos). Dadas las limitaciones del tamaño muestral, las personas ubicadas en una posición de clase "capitalista" (empleadores con 10 o más empleados) fueron integradas en una categoría de clase separada que también incluyó directivos expertos de alto nivel. Esta categoría de "empresarios y directivos expertos" fue construida para representar mejor a las personas ubicadas en posiciones de clase "privilegiadas" en las relaciones de explotación y dominación en el ámbito productivo.

Siguiendo a Wright (1997), dentro de la población asalariada se diferenció a las posiciones de clase de acuerdo con la posesión de cualificaciones/credenciales y bienes de organización (autoridad). A partir de la Clasificación Internacional Uniforme de Ocupaciones (CIUO-1988, dos dígitos) y del nivel educacional, se identificaron tres categorías de asalariados según cualificaciones y/o credenciales: expertos, trabajadores calificados y trabajadores no calificados. De modo similar, en base a una pregunta del cuestionario destinada a determinar si el encuestado ocupaba un puesto de supervisión en su lugar de trabajo, se distinguió a los trabajadores según su posición de autoridad (tiene / no tiene autoridad). Aunque esta distinción es ciertamente una simplificación de la dimensión de autoridad propuesta en el modelo de Wright (1997), ella permitió, al ser combinada con los niveles de calificación/credenciales, identificar distintas "posiciones contradictorias de clase" tales como expertos sin autoridad y supervisores calificados, así como dos categorías que denotan a la clase trabajadora calificada y no calificada. 
Finalmente, combinando las clases propietarias y asalariadas, se construyó una variable compuesta por nueve categorías de clases (porcentajes se presentan entre paréntesis): Empresarios y directivos expertos $(6,6 \%)$; Pequeños empleadores $(4,6 \%)$; Pequeña burguesía formal (3,1\%); Expertos sin autoridad (4,6\%); Supervisores calificados (8,2\%); Supervisores no calificados (9,8\%); Trabajadores calificados (17,5\%); Trabajadores no calificados (34,6\%); y Autoempleados informales $(11,2 \%)$.

Junto con la posición objetiva de clase, la clase social fue medida a partir de la autoidentificación de clase. Esta variable fue construida a partir de la pregunta: "En nuestra sociedad hay grupos que tienden a ubicarse en los niveles más bajos y en los niveles más altos de la sociedad. ¿Dónde se ubicaría usted?". Las respuestas, originalmente medidas en una escala de 1 a 10, fueron recodificadas en tres categorías (porcentajes entre paréntesis): clase baja (26,5\%); clase media (52,9\%); y clase alta $(20,65 \%)$.

\section{Percepción de privación económica}

Considerando la manera en que esta variable ha sido medida en investigaciones previas (Hadler, 2005; Robinson y Bell, 1978), en este artículo operacionalizamos la percepción de privación a partir de la pregunta: "Considerando el dinero que recibe mensualmente, ¿usted piensa que es menos de lo que merece, está bien, o es más de lo que se merece?". Las opciones de respuesta variaron entre 1 (Mucho menos de lo que merezco) y 5 (Mucho más de lo que merezco). Sin embargo, debido a que muy pocos encuestados señalaron recibir "mucho más" de lo que merecían, esta variable fue recodificada de la siguiente manera (porcentajes entre paréntesis): 1 = Recibo el ingreso mensual que merezco o más (38\%); 2 $=$ Recibo menos ingreso mensual del que merezco (40,1\%); y 3 = Recibo mucho menos ingreso mensual del que merezco $(21,9 \%)$.

En algunas investigaciones la percepción de privación económica ha sido operacionalizada a través de otras preguntas de cuestionario. Por ejemplo, Kreidl (2000) utiliza una variable que mide si los encuestados consideran que el ingreso que ganan les alcanza para cubrir las necesidades del hogar. A fin de chequear si la variable que utilizamos acá afectó los resultados, desarrollamos los mismos análisis estadísticos presentados en la sección siguiente, pero usando una variable que se acerca en cierto modo a la ocupada por Kreidl. Esta variable fue 
el resultado de la pregunta "¿Cómo calificaría en general su situación económica actual y la de su familia?" (1. Mala; 2 . Ni buena ni mala; 3 . Buena). Los resultados de estos análisis, disponibles por solicitud, no mostraron diferencias sustanciales a los acá presentados.

\section{Identificación política}

Esta variable fue construida a partir de la pregunta: "En una escala de 1 a 10, donde 1 representa más de izquierda y 10 más de derecha, ¿en qué posición se ubicaría usted?". A fin de mantener los casos que no se identificaron con el continuo izquierda-derecha de la escala y que escogieron la opción "no sabe / no responde", esta variable fue recodificada en cuatro categorías (porcentajes entre paréntesis): Izquierda (23,3\%); Centro (35,6\%); Derecha (13,55\%); Sin identificación (27,5\%).

Para testear las hipótesis señaladas anteriormente, estas variables independientes fueron introducidas en cinco modelos de regresión de mínimos cuadrados ordinarios (regresiones MCO). Mientras los primeros tres modelos incluyen solo las variables independientes de interés, el modelo 4 muestra los coeficientes de todas estas variables introducidas conjuntamente. Finalmente, en el modelo 5 se incluyen tres controles sociodemográficos utilizados comúnmente en estudios similares (Robison y Stubager, 2018): edad (en años); sexo ( $1=$ femenino, $0=$ masculino); y estado civil ( $1=$ casado o conviviente, $0=$ otro $)$. Análisis estadísticos previos sugirieron que ninguno de estos modelos presentó problemas de multicolinealidad (todos los predictores incluidos en estos tuvieron un VIF inferior a 3,5).

\section{Resultados}

La Tabla 2 muestra los resultados (coeficientes no estandarizados) de los modelos de regresión de mínimos cuadrados ordinarios. Los modelos indican, tal como se planteó en la $\mathrm{H}_{1.1}$, que existe una relación significativa entre posición de clase y percepción del conflicto entre clases. Quienes se encuentran en una posición subordinada en las relaciones de explotación (clase trabajadora calificada y no calificada y clase de autoempleados informales) perciben mayores niveles de oposición de clases que quienes se encuentran en una posición privilegiada (empresarios y directivos expertos) (modelo 1). Los modelos de regresión también sugieren que las personas pertenecientes a posiciones de clase contradictorias como la de 
los supervisores no calificados, poseen percepciones del conflicto de clases significativamente mayores que quienes pertenecen a la categoría de referencia. Algo similar ocurre, incluso, con quienes pertenecen a la pequeña burguesía formal. Sin embargo, el coeficiente para esta última no es estadísticamente significativo según los criterios convencionales (valor- $p<0,05$ ), probablemente debido al bajo número de casos ubicados en esta categoría de clase. Cabe mencionar que el efecto de la posición de clase no es, en general, sustancialmente grande en magnitud. Por ejemplo, ser un trabajador o trabajadora no calificada conlleva un incremento promedio de poco más de 1 punto de la escala de percepción del conflicto de clases, en comparación con ser un empresario o un directivo experto (modelo 1). Sin embargo, dicho efecto es estadísticamente robusto y se mantiene significativo incluso cuando es controlado por todas las otras variables incluidas en los modelos 4 y 5 .

Tal como la posición objetiva de clase, la autoidentificación de clase tiene un efecto significativo sobre la variable dependiente. Como planeta la $\mathrm{H}_{1.2}$, quienes se identifican como parte de la clase baja - e incluso, aunque en menor medida, de la clase media - perciben más conflicto entre trabajadores y empresarios que quienes se identifican con la clase alta (modelo 1). El impacto de la autoidentificación se mantiene robusto incluso en los modelos donde se incluyen las otras variables independientes o de control (modelos 4 y 5 respectivamente).

En relación con la $\mathrm{H}_{2}$, la cual afirma una relación significativa entre las percepciones de privación económica y de conflicto de clases percibido, los resultados indican que ella debe ser aceptada. A mayor privación económica percibida, mayores percepciones de conflicto. Así, por ejemplo, quienes sienten que reciben "mucho menos" de lo que merecen, tienen un puntaje promedio casi 2 puntos más altos $(1,887$ puntos, según el modelo 5) que quienes piensan que reciben lo que merecen o más (valor- $p<0,001$ ). Tal como en el caso de las variables de clase, el efecto de la privación económica se mantiene robusto incluso cuando se agregan controles sociodemográficos (modelo 5). La importancia de esta variable también se puede ver en el $\mathrm{R}^{2}$ ajustado. Según el modelo 2, dicha variable explica, por sí sola, casi el 9\% de las variaciones de la variable dependiente. Esto es alto si se considera que las variables de clase y de identificación política explican, respectivamente, $4 \%$ y $5 \%$ de la varianza de la variable dependiente (modelos 1 y 3 ). 
Por último, respecto de la relación entre posición política y percepción de conflicto de clases, los coeficientes sugieren que la $\mathrm{H}_{3}$ también debe ser aceptada. Tanto el modelo 3 como los modelos con más controles (modelos 4 y 5 ) indican que posicionarse en la derecha implica una disminución promedio de poco más de 1 punto en la escala de percepción del conflicto de clase, en comparación a posicionarse en la izquierda (valor- $p<0,001$ ). Los modelos también sugieren que existe una diferencia menor, pero estadísticamente significativa, entre posicionarse en el centro y en la izquierda (quienes se posicionan en el centro político perciben menos oposición de clase). Por último, los coeficientes indican que no existen diferencias significativas entre quienes se identifican con la izquierda y quienes no se identifican con el continuo izquierdaderecha. Esto implica que en estos dos grupos de identificación política se concentran las mayores percepciones de conflicto.

Tabla 2

Determinantes de la percepción del conflicto de clases en Chile, 2014 (modelos de regresión $M C O$, coeficientes no estandarizados, errores estándares entre paréntesis)

\begin{tabular}{|c|c|c|c|c|c|}
\hline & $\begin{array}{c}\text { Modelo } \\
1\end{array}$ & $\begin{array}{c}\text { Modelo } \\
2\end{array}$ & $\begin{array}{c}\text { Modelo } \\
3\end{array}$ & $\begin{array}{c}\text { Modelo } \\
4\end{array}$ & Modelo 5 \\
\hline \multicolumn{6}{|l|}{$\begin{array}{l}\text { Posición de clase (Ref.: } \\
\text { empresarios y ejecutivos } \\
\text { expertos) }\end{array}$} \\
\hline \multirow[t]{2}{*}{ 2. Pequeños empresarios } & 0,726 & & & 0,597 & 0,608 \\
\hline & $(0,607)$ & & & $(0,586)$ & $(0,587)$ \\
\hline \multirow[t]{2}{*}{ 3. Pequeña burguesía formal } & 1,192 & & & 1,245 & 1,278 \\
\hline & $(0,687)$ & & & $(0,661)$ & $(0,663)$ \\
\hline \multirow[t]{2}{*}{ 4. Expertos } & 0,591 & & & 0,309 & 0,322 \\
\hline & $(0,607)$ & & & $(0,586)$ & $(0,588)$ \\
\hline \multirow[t]{2}{*}{ 5. Supervisores calificados } & 0,686 & & & 0,551 & 0,618 \\
\hline & $(0,527)$ & & & $(0,507)$ & $(0,510)$ \\
\hline \multirow[t]{2}{*}{ 6. Supervisores no calificados } & $1,330^{* *}$ & & & $1,170^{*}$ & $1,196^{*}$ \\
\hline & $(0,510)$ & & & $(0,490)$ & $(0,492)$ \\
\hline \multirow[t]{2}{*}{ 7. Trabajadores calificados } & $1,109^{*}$ & & & $0,975^{*}$ & $1,014^{*}$ \\
\hline & $(0,460)$ & & & $(0,443)$ & $(0,445)$ \\
\hline \multirow[t]{2}{*}{ 8. Trabajadores no calificados } & $1,150^{* *}$ & & & $0,955^{*}$ & $0,976^{*}$ \\
\hline & $(0,431)$ & & & $(0,416)$ & $(0,418)$ \\
\hline \multirow[t]{2}{*}{ 9. Autoempleados informales } & $1,142^{*}$ & & & $0,995^{*}$ & $0,955^{*}$ \\
\hline & $(0,494)$ & & & $(0,477)$ & $(0,478)$ \\
\hline
\end{tabular}




\begin{tabular}{lccc}
\hline $\begin{array}{l}\text { Autoidentificación de clase (Ref: } \\
\text { clase media alta y alta) }\end{array}$ & & & \\
\hline 1. Clase baja & $1,726^{* * *}$ & $0,801^{* * *}$ & $0,766^{*}$ \\
\hline & $(0,302)$ & $(0,308)$ & $(0,310)$ \\
\hline & $0,804^{* *}$ & 0,266 & 0,236 \\
\hline 2. Clase media & $(0,263)$ & $(0,261)$ & $(0,262)$ \\
\hline $\begin{array}{l}\text { Privación económica (Ref: } \\
\text { recibo lo que merezco o más) }\end{array}$ & & & \\
\hline $\begin{array}{l}\text { 2. Recibo menos de lo que } \\
\text { merezco }\end{array}$ & $1,281^{* * *}$ & $0,918^{* * *}$ & $0,932^{* * *}$ \\
\hline & $(0,221)$ & $(0,228)$ & $(0,228)$ \\
\hline $\begin{array}{l}\text { 3. Recibo mucho menos de lo } \\
\text { que merezco }\end{array}$ & $2,410^{* * *}$ & $1,890^{* * *}$ & $1,887^{* * *}$ \\
\hline & $(0,262)$ & $(0,277)$ & $(0,277)$ \\
\hline
\end{tabular}

Identificación política (Ref.:

izquierda)

\begin{tabular}{lccc}
\hline 2. Centro & $-0,825^{* *}$ & $-0,620^{*}$ & $-0,598^{*}$ \\
\hline 3. Derecha & $(0,266)$ & $(0,261)$ & $(0,262)$ \\
\hline & $-1,583^{* * *}$ & $-1,196^{* * *}$ & $-1,213^{* * *}$ \\
\hline 4. Sin identificación & $(0,341)$ & $(0,335)$ & $(0,336)$ \\
\hline & 0,405 & 0,166 & 0,181 \\
\hline & $(0,281)$ & $(0,273)$ & $(0,274)$ \\
\hline
\end{tabular}

Controles sociodemográficos

\begin{tabular}{lc}
\hline Edad & 0,00526 \\
\hline Género (Ref:: hombre) & $(0,00794)$ \\
\hline & 0,206 \\
\hline
\end{tabular}

Estado civil (Ref.: no casado ni

$\begin{array}{ll}\text { conviviente) } & 0,161\end{array}$

$(0,202)$

\begin{tabular}{cccccc}
\hline Constante & $10,57^{* * *}$ & $11,41^{* * *}$ & $12,85^{* * *}$ & $10,81^{* * *}$ & $10,38^{* * *}$ \\
\hline & $(0,421)$ & $(0,159)$ & $(0,207)$ & $(0,456)$ & $(0,592)$ \\
\hline
\end{tabular}

\begin{tabular}{lccccc}
\hline $\mathrm{N}$ & 930 & 930 & 930 & 930 & 930 \\
\hline R cuadrado ajustado & 0,04 & 0,09 & 0,05 & 0,12 & 0,12 \\
\hline
\end{tabular}

Errores estándares entre paréntesis

${ }^{* * *} p<0,001 ;{ }^{* *} p<0,01 ;{ }^{*} p<0,05$

Fuente: Elaboración propia. 


\section{Discusión}

En línea con la literatura reciente (Elbert y Pérez, 2018; Hout, 2008; Robison y Stubager, 2018; Wright, 2015), los modelos de regresión indican que, tanto en términos de posición "objetiva" como de realidad subjetivamente expresada, la clase social es un predictor significativo de los intereses antagónicos de los chilenos. Esto contradice la hipótesis del "fin de las clases" (Pakulski y Waters, 1996) y, en el caso de Chile, los análisis que afirman que el vínculo entre la posición objetiva de clase y las experiencias subjetivas se ha debilitado (Araujo y Martuccelli, 2011; Arteaga, 2017). Por ejemplo, se señala que los discursos neoliberales han permeado de otra manera a todas las clases sociales (Arteaga, 2017), lo cual implica, según este argumento, que las experiencias de desigualdad que enfrentan las personas y las estrategias que utilizan para lidiar con ellas no difieren sustancialmente de una clase a otra. Sin desestimar el hecho de que las experiencias de desigualdad en Chile son experimentadas por segmentos amplios de la población, nuestros hallazgos revelan que, al menos en lo que se refiere a las percepciones de la oposición de clase, la clase social sigue siendo importante para distinguir la intensidad con que se percibe dicha oposición.

Un aspecto que merece ser destacado es el impacto que tienen las percepciones de privación económica. La literatura internacional ha demostrado que dichas percepciones representan un mecanismo central a través del cual la desigualdad socioeconómica de clases se manifiesta a nivel subjetivo (Hadler, 2005; Kreidl, 2000; Robinson y Bell, 1978; Szirmai, 1986). Tal como esta literatura, la evidencia presentada acá sugiere que el nivel de privación económica percibido afecta de modo significativo la percepción del conflicto de clases. Más aún, el análisis separado de cada variable independiente demostró que la privación económica es la que tiene más impacto sobre la variable dependiente. En la medida en que los sentimientos de privación económica representan una manifestación subjetiva de desventajas de clase objetivas, ellos son un factor que refuerza de manera significativa los intereses antiempresariales medidos en la escala de conflicto de clases utilizada como variable dependiente. Es probable que este tipo de factores tenga un impacto importante en los fenómenos de protesta observados después del año en que fueron obtenidos los datos acá analizados (2014). En efecto, análisis recientes sostienen que factores 
subjetivos de este tipo son los que han estado detrás del crecimiento de la protesta social en Chile (Joignant et al., 2017; Luna, 2016; PNUD, 2015; Somma, 2017). Más aún, las demandas que dieron vida al ciclo de movilizaciones iniciadas en octubre de 2019 - aumento de salario mínimo, desmercantilización de derechos sociales, etc. (Santibáñez, 2019)-, indican que esos mismos sentimientos de privación económica aún siguen operando como mecanismo subjetivo que facilita la acción colectiva.

Por último, los resultados de los modelos de regresión también contribuyen a entender de mejor modo los efectos subjetivos del reforzamiento del clivaje izquierda-derecha notado recientemente por algunos autores (Donoso y Von Bülow, 2017; PNUD, 2015; Roberts, 2017). Los datos analizados indican, en efecto, que la distinción izquierdaderecha, medida según el autoposicionamiento de los encuestados, sigue siendo relevante para entender las percepciones de conflicto social. Esto supone matizar los resultados de investigaciones en donde se ha argumentado que dicho clivaje ha perdido relevancia en Chile.

Sin embargo, se debe notar que el distanciamiento entre partidos políticos y la ciudadanía sigue siendo muy fuerte en Chile. Si bien los eventos de protestas de los últimos años han servido para "politizar la desigualdad", ellos se han desarrollado en gran medida al margen de los partidos tradicionales. Esto podría ayudar a explicar por qué, según indican los modelos de regresión, no existen diferencias significativas en el conflicto percibido por quienes se posicionan en la izquierda y por quienes declaran que no tienen identificación política alguna (más aún, los modelos sugieren que estos últimos podrían incluso percibir más oposición de clase). Si se utiliza la escala de percepción de conflicto de clases como indicador de actitudes "críticas" hacia la desigualdad en Chile, estos resultados sugieren que al menos una parte importante de quienes se movilizan actualmente no lo hacen por cuestiones político-ideológicas, sino que probablemente como resultado de un malestar social "no politizado", al menos en el sentido de no identificarse con las categorías tradicionales de izquierda, centro y derecha.

\section{Conclusión}

Los hallazgos de este artículo permiten concluir que, contrario a lo planteado por la tesis del "fin de las clases", la clase social tiene impor- 
tancia no solo en términos objetivos, sino que también como realidad subjetiva. Esta realidad subjetiva se expresa tanto en la identificación con una clase en particular, como en percepciones de privación económica que refuerzan la conciencia del conflicto entre trabajadores y empresarios. Los resultados presentados también demuestran la importancia que tiene la identificación con el clivaje izquierda-derecha al momento de explicar cómo se percibe el conflicto de clases. A pesar de esto, los modelos de regresión sugieren que, en un contexto de fuerte separación entre partidos políticos y movimientos sociales, la conciencia de la oposición de clases también puede reforzarse por factores no circunscritos al continuo izquierda-derecha (se debe recordar que quienes no se identifican con dicho continuo tienen percepciones del conflicto tan altas como quienes se identifican con la izquierda).

Si bien los datos analizados en este trabajo fueron recolectados en 2014, creemos que nuestros hallazgos son útiles para entender de mejor forma porqué los chilenos se tomaron las calles del país en octubre de 2019 demandando el congelamiento de las tarifas de transporte público, mejoras sustanciales en las condiciones de vida y, de modo más general, cambios profundos al modelo neoliberal. Tanto las demandas de los manifestantes en las calles como el pliego de peticiones de la coordinadora de movimientos sociales que articuló los llamados a huelga general de noviembre 2019 (Unidad Social) sugieren que los factores políticos y de clase destacados en este artículo jugaron un rol central.

En primer lugar, todas las demandas que dieron vida a la movilización tuvieron un fuerte componente de clase derivado de los altos niveles desigualdad socioeconómica y de poder existentes en el país (Santibáñez, 2019). En segundo lugar, los eventos recientes también indican la importancia de factores políticos y, al mismo tiempo, el carácter en cierto modo paradójico del proceso de politización observado en Chile. En efecto, gran parte de los discursos que sustentaron las movilizaciones de fines de 2019 reflejan una continuidad importante con las demandas de los movimientos sociales de 2011. Un ejemplo claro de esto es la oposición entre "neoliberalismo" y "derechos sociales" sostenida por quienes exigen el fin del modelo neoliberal y promueven, desde la Convención Constitucional y desde las calles, una nueva constitución que permita superar la herencia institucional de 
la dictadura. Esto refuerza la idea de que la sociedad chilena ha experimentado un proceso de politización que, en la medida en que se funda en una politización de clivajes socioeconómicos, probablemente se traduzca en un reforzamiento de la división izquierda-derecha en el país. Sin embargo, es importante notar que dicha politización sigue ocurriendo al margen de los partidos tradicionales (con excepción, tal vez, del Partido Comunista). Hasta el día de hoy, dichos partidos no parecen ser capaces de responder a las demandas ciudadanas, más allá de proponer los tradicionales acuerdos "entre élites" tan comunes en los años 1990 y 2000.

Aún es temprano para evaluar los efectos concretos de las movilizaciones de 2019, más aún cuando la pandemia mundial por COVID-19 interrumpió el debate político y social resultante de ellas. Sin embargo, en lo que respecta al argumento desarrollado en este artículo, parece ser claro que la explosión de la movilización social observada desde octubre de 2019 en Chile fue una expresión más de un proceso de malestar y politización que se ha venido incubando por años. Si se logra redactar una nueva constitución con verdadera participación popular y si los partidos de izquierda, tradicionales o nuevos, deciden reconstruir los lazos con las organizaciones sociales de base, es probable que Chile pase a nueva etapa de la política de clases. Es esta nueva etapa, el conflicto observado a nivel subjetivo en la polarización de actitudes podría traducirse en mayor poder de movilización por parte de la clase trabajadora. Ello podría implicar, a su vez, pasar a una fase en donde la clase trabajadora y los sectores populares se movilicen no solo para recuperar lo que les fue arrebatado décadas atrás, sino también para construir un nuevo modelo de sociedad en donde el poder económico y político pueda ser democratizado en beneficio de todos los chilenos y chilenas.

\section{Referencias}

Alesina, A. y Giuliano, P. (2011). Preferences for redistribution. En A. Bisin y J. Benhabib (Eds.), Handbook of social economics (pp. 93-132). North Holland.

Andersen, R. y Curtis, J. (2012). The polarizing effect of economic inequality on class identification: Evidence from 44 countries. Research in Social Stratification and Mobility, 30(1), 129-141. https://doi.org/10.1016/j.rssm.2012.01.002 
Angell, A. (1972). Politics and the labour movement in Chile. Royal Institute of International Affairs.

Araujo, K. y Martuccelli, D. (2011). La inconsistencia posicional: Un nuevo concepto sobre la estratificación social. Revista de la CEPAL, (103), 165-178. http://hdl.handle.net/11362/11453

Arteaga,C.(2017).Diversidaddeexperienciasposicionalesenelcontexto de las transformaciones en la estructura social chilena. Sociológica, (32), 1-32. http://www.scielo.org.mx/scielo. php?script=sci_arttext $\&$ pid=S0187-01732017000200045

Burak, E. (2013). The social maximum: American attitudes toward extremely high incomes. Research in Social Stratification and Mobility, (31), 97-114. https://doi.org/10.1016/j. rssm.2012.11.003

Castillo, J. C., Bargsted, M., Olivos, F., y Mackenna, B. (2015). Percepción del conflicto en Chile: Un análisis desde la opinión pública, 2006-2013. En M. Castillo y C. Maldonado (Eds.), Desigualdades. Tolerancia, legitimación y conflicto en las sociedades latinoamericanas. RIL Editores.

Castillo, J. C., Madero-Cabib, I., y Salamovich, A. (2013). Clivajes partidarios y cambios en preferencias distributivas en Chile. Revista de Ciencia Política (Santiago), 33(2), 469-488. https://doi.org/10.4067/S0718-090X2013000200003

COES (2014). Encuesta Nacional COES. Centro de Estudios del Conflicto y Cohesión Social. Santiago de Chile.

Cortés, A. (mayo de 20 de 2021). Estallido electoral. El Mostrador. https://www.elmostrador.cl/destacado/2021/05/20/ estallido-electoral/

Dodson, K. (2017). Economic change and class conflict over tax attitudes: Evidence from nine advanced capitalist democracies. Social Forces, 95(4), 1509-1538. https://doi. org/10.1093/SF\%2FSOX027

Donoso, S. (2016). When social movements become a democratizing force: The political impact of the student movement in Chile. En T. Davies (Ed.), Protest, social movements, and global democracy since 2011: New perspectives (pp. 167-196). Emerald Group Publishing Limited.

Donoso, S. y Von Bülow, M. (2017). Introduction: Social movements in contemporary Chile. En S. Donoso y Von Bülow (Eds.), Social 
movements in Chile: Organization, trajectories, and political consequences (pp. 3-28). Palgrave Macmillan.

Drake, P. W. (1996). Labor movements and dictatorships: The Southern Cone in comparative perspective. Johns Hopkins University Press.

Edlund, J. y Lindh, A. (2015). The democratic class struggle revisited: The welfare state, social cohesion, and political conflict. Acta Sociológica, 58(4), 311-328. https://doi. org/10.1177/0001699315610176

Elbert, R. y Pérez, P. (2018). The identity of class in Latin America: Objective class position and subjective class identification in Argentina and Chile (2009). Current Sociology, 66(5), 724747. https://doi.org/10.1177/0011392117749685

Evans, G. (1992). Is Britain a class-divided society? A re-analysis and extension of Marshall et al.'s study of class consciousness. Sociology, 26(2), 233-258. https://doi.org/10.1177/00380385 92026002007

Evans, G. (1997). Political ideology and popular beliefs about class and opportunity: Evidence from a survey experiment. British Journal of Sociology, 48(3), 450-470. https://doi. org $/ 10.2307 / 591140$

Fantasia, R. (1988). Cultures of solidarity: Consciousness, action, and contemporary American workers. University of California Press.

Garretón, M. A. (2016). La gran ruptura: Institucionalidad política y actores sociales en el Chile del siglo XXI. LOM Ediciones.

Hadler, M. (2005). Why do people accept different income ratios? A Multi-level Comparison of Thirty Countries. Acta Sociológica, 48(2), 131-154. https://doi.org/10.1177/0001699305053768

Hout, M. (2008). How class works: Objective and subjective aspects of class salience since the 1970s. En A. Lareau y D. Conley (Eds.), Social class: How does it work? (pp. 25-64). Russell Sage Foundation.

Huber, E. y Stephens, J. D. (2001). Development and crisis of the welfare state: Parties and Policies in Global Markets. University of Chicago Press.

Joignant, A., Morales, M., y Fuentes, C. (2017). Malaise in representation: Attitudes, Beliefs, behaviors, and causalities. En A. Joignant, M. Morales, y C. Fuentes (Eds.), Malaise in representation 
in Latin American countries: Chile, Argentina, and Uruguay (pp. 1-43). Palgrave Macmillan US.

Kelley, J. y Evans, M. D. (1995). Class and class conflict in six Western nations. American Sociological Review, 60(2), 157-178. https://doi.org/10.2307/2096382

Kelley, J. y Evans, M. D. R. (2017). Societal inequality and individual subjective well-being: Results from 68 societies and over 200,000 individuals, 1981-2008. Social Science Research, (62), 1-23. https://doi.org/10.1016/j.ssresearch.2016.04.020

Kingstone, P., Young, J. K., y Aubrey, R. (2013). Resistance to privatization: Why protest movements succeed and fail in Latin America. Latin American Politics and Society, 55(3), 93116. https://doi.org/10.1111/j.1548-2456.2013.00204.x

Kluegel, J. R. y Smith, E. R. (1986). Beliefs about inequality: Americans' views of what is and what ought to be. Aldine de Gruyter.

Korpi, W. (1985). Power resources approach vs. action and conflict: On causal and intentional explanations in the study of power. Sociological Theory, 3(2), 31-45. https://doi. org/10.2307/202223

Kreidl, M. (2000). Perceptions of poverty and wealth in Western and post-communist countries. Social Justice Research, 13(2), 151-176. https://doi.org/10.1023/A:1007597807110

Langsæther, P. E. y Evans, G. (2020). More than self-interest: Why different classes have different attitudes to income inequality. The British Journal of Sociology, 71(4), 594-607. https://doi. org/10.1111/1468-4446.12747

Latinobarómetro (2021). Bases de dato corporación Latinobarómetro. Disponible en https://www.latinobarometro.org/lat.jsp

Luna, J. P. (2016). Delegative democracy revisited: Chile's crisis of representation. Journal of Democracy, 27(3), 129-138. https://www.journalofdemocracy.org/articles/delegativedemocracy-revisited-chiles-crisis-of-representation/

Madrid, R. (2003). Labouring against neoliberalism: Unions and patterns of reform in Latin America. Journal of Latin American Studies, 35(1), 53-88. https://doi.org/10.1017/ s0022216x0200665x

Murillo, M. V. (2001). Labor unions, partisan coalitions, and market reforms in Latin America. Cambridge University Press. 
Oddsson, G. (2018). Class imagery and subjective social location during Iceland's economic crisis, 2008-2010. Sociological Focus, 51(1), 14-30. https://doi.org/10.1080/00380237.2017.1341251

Pakulski, J. y Waters, M. (1996). The death of class. Sage.

Pérez, P. (2014). Class consciousness in a mature neoliberal society: Evidence from Chile. Research in Social Stratification and Mobility, (38), 57-75. https://doi.org/10.1016/j. rssm.2014.06.002

Pérez, P. (2018). Clases sociales, sectores económicos y cambios en la estructura social chilena (1992-2013). Revista de la CEPAL, (126), 171-192. https://www.cepal.org/es/ publicaciones/44308-clases-sociales-sectores-economicoscambios-la-estructura-social-chilena-1992

Programa de las Naciones Unidas para el Desarrollo, PNUD. (2015). Desarrollo humano en Chile: Los tiempos de la politización. PNUD.

Reynolds, J. y Xian, H. (2014). Perceptions of meritocracy in the land of opportunity. Research in Social Stratification and Mobility, (36), 121-137. https://doi.org/10.1016/j.rssm.2014.03.001

Roberts, K. M. (2017). Chilean social movements and party politics in comparative perspective: Conceptualizing Latin America's "Third generation" of anti-neoliberal protest. En M. von Bülow y S. Donoso (Eds.), Social movements in Chile: Organization, trajectories, and political consequences (pp. 221-247). Palgrave Macmillan.

Robinson, R. V. y Bell, W. (1978). Equality, success, and social justice in England and the United States. American Sociological Review, 43(2), 125-143. https://doi.org/10.2307/2094695

Robison, J. y Stubager, R. (2018). The class pictures in citizens' minds. The British Journal of Sociology, 69(4), 1220-1247. https:// doi.org/10.1111/1468-4446.12313

Santibáñez, C. (2019). "It's not about 30 pesos; It's about 30 years": The working-class revolt in the laboratory of neoliberalism. Labor Notes. https://www.labornotes.org/2019/11/chile-revolt-itsnot-about-30-pesos-its-about-30-years

Somma, N. (2017). Discontent, collective protest, and social movements in Chile. En A. Joignant, M. Morales, y C. Fuentes (Eds.), Malaise in representation in Latin American countries: Chile, Argentina, and Uruguay (pp. 47-68). Palgrave Macmillan US. 
Somma, N. M., Bargsted, M., Pavlic, R. D., y Medel, R. M. (2020). No water in the oasis: The Chilean spring of 2019-2020. Social Movement Studies, 20(4), 495-502. https://doi.org/10.1080/ 14742837.2020.1727737

Somma, N. M. y Medel, R. (2017). Shifting relationships between social movements and institutional politics. En M. von Bülow y S. Donoso (Eds.), Social movements in Chile: Organization, trajectories, and political consequences (pp. 29-61). Palgrave Macmillan.

Svallfors, S. (2006). The moral economy of class: Class and attitudes in comparative perspective. Stanford University Press.

Szirmai, A. (1986). Inequality observed: A study of attitudes towards income inequality. Aversbury.

Thompson, E. P. (1966). The making of the English working class. Vintage Books.

Unidad Social. (2019). Las urgencias para transformar Chile: Pliego de demandas. https://cut.cl/cutchile/2019/12/09/pliego-dedemandas-de-unidad-social-comprende-16-ambitos-deaccion/

Vanneman, R. y Cannon, L. W. (1987). The American perception of class. Temple University Press.

Weakliem, D. L. y Adams, J. (2011). What do we mean by "class politics"? Politics \& Society, 39(4), 475-495. https://doi. org/10.1177/0032329211420047

Wright, E. O. (1989). Rethinking, once again, the concept of class structure. En E. O. Wright (Ed.), The debate on classes. Verso.

Wright, E. O. (1997). Class counts: Comparative studies in class analysis. Cambridge University Press.

Wright, E. O. (2015). Understanding class. Verso. 